Drug Resistance

\title{
A SURVEY OF METHICILLIN RESISTANCE IN STAPHYLOCOCCUS AUREUS
}

\author{
M. T. Parker and M. Patricia Jevons \\ Cross-Infection Reference Laboratory, Colindale, London, N.W.9.
}

IN an earlier symposium on the resistance of bacteria to the penicillins, Barber (1962) emphasized the difficulties of obtaining an estimate of the incidence of methicillin resistance in Staphylococcus aureus. To yield reliable information, the population under investigation must be large and diverse, and must be sampled in a uniform way; and the degree of resistance to be recorded must be defined precisely. In the survey we began in 1960 we have tried to fulfil these criteria.

During the last three and a half years we have carried out tests for methicillin resistance on 43,867 of the 60,053 cultures of Staph. aureus we received for typing. The way in which they were selected has already been described (Jevons, Coe and Parker, 1963). Most of them came from hospitals in south-eastern England and were isolated from hospital patients and staff, but we excluded all cultures sent to us because they had been found to be methicillin resistant.

We used a screening test in which filterpaper discs containing $10 \mu \mathrm{g}$. methicillin were placed on plates inoculated from 6-hour broth cultures. Cultures naturally resistant to methicillin appear to consist of a minority of individuals with a high resistance and a majority whose resistance is only slightly increased (Knox, 1961; Knox and Smith, 1961; Sutherland and Rolinson, 1964). The appearance of a disc-sensitivity test on a resistant culture depends, therefore, on the proportion of highly resistant individuals it contains. Some cultures grow continuously up to the disc, but others show no more than a slight narrowing of the zone of inhibition with a few discrete colonies nearer to the disc (Fig. 1).

Cultures which were apparently resistant to methicillin when tested with discs were also tested by a tube-dilution method, and were finally regarded as resistant only if the minimum inhibitory concentration (M.I.C.) was $12.5 \mu \mathrm{g}$. methicillin $/ \mathrm{ml}$. or more after 18 hours incubation. (For full details see Jevons et al., 1963).

\section{Incidence}

In the three months October to December 1960 we examined 5,440 cultures of Staph aureus and found three that were methicillip resistant, all from one hospital (Jevons, 1961) One of the resistant organisms came from as patient from whose nose and skin Staph. aureus? had previously been isolated in July, $1960 \%$ Fortunately the earlier cultures had been kept. They were found also to be methicillino resistant, though the M.I.C. for methicillin was lower than that of the organisms isolated. later. Methicillin was not used in this hospitat before October, 1960. Several other accounts have been published of the appearance of methicillin-resistant Staph. aureus in place where methicillin had not been used (for example, Cetin and Ang, 1962; Borowšis: Kamiénska and Rutecka, 1964). It is likey therefore, that the origin of naturally methi cillin-resistant strains of Staph. aureus is unconnected with the use of methicillin.

Since our first survey (Jevons, 1961), wळ̈ examined several thousand cultures in each subsequent quarter-year. During 1961 and 1962 there was a slow increase in the propor 3 tion of resistant cultures, but their incidence appears to have changed little since the middle of 1962 (Table I). Our figures for 1961 and 1962 were, however, rather over-weighted by the inclusion of a large number of resistan cultures from Queen Mary's Hospital fo Children, Carshalton, where an exhaustive search for resistant organisms was being made. If the Carshalton cultures are excluded we have a truer estimate of changes in the incidence of resistance. Fig. 2 shows that, up to the middle of 1962, the percentage of resistanto cultures (excluding Carshalton) fluctuated between 0.06 and 0.29 . In the third quarter of that year it rose to 0.61 and has remained ate
about that level since.

Table II shows the total number of culturese which came from each of the laboratories submitting methicillin-resistant staphylococci, and the number from each source that were resist- 0 


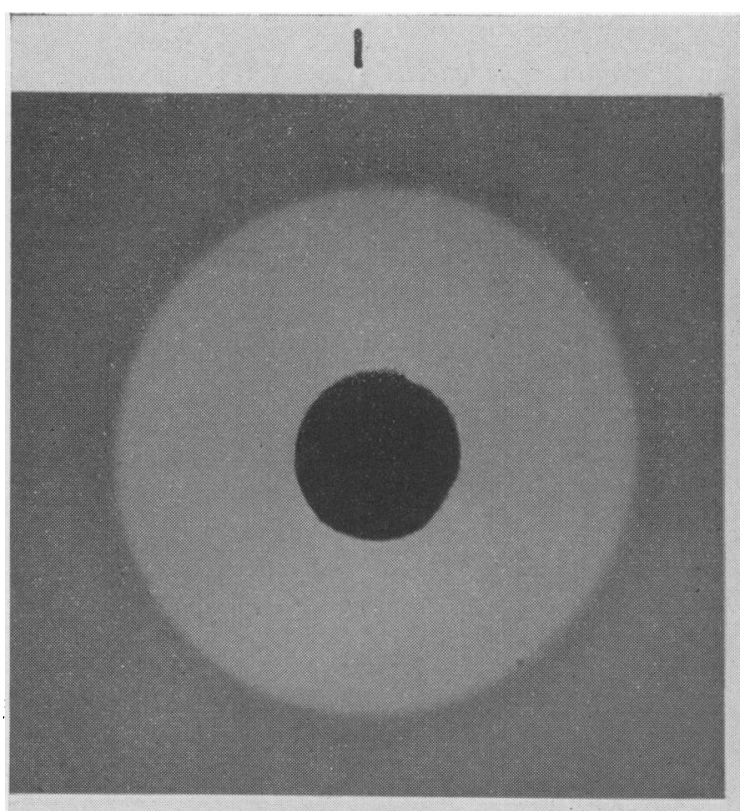

3

FIG. 1.-Disc sensitivity tests with methicillin. Each disc contains $10 \mu \mathrm{g}$. methicillin. (1) methicillin-sensitive culture, (2), (3) and (4) resistant cultures (see text).

ant in each three-month period. Only one resistant culture from each infected person was included. Resistant organisms were received from 35 laboratories. Their sources included 31 different British hospitals, three foreign hospitals, and the Food Hygiene Laboratory at Colindale. A total of 198 hospitals in Great Britain sent us cultures, and one or more methicillin-resistant culture was obtained from
$16 \%$ of them. The number of resistant cultures from one hospital was seldom large, and was in most cases a very small proportion of all the cultures sent. Indeed, there was a close correlation between the total number of cultures submitted and the chance that they would include a resistant organism. In the first two years of our survey (Jevons et al., 1963), no resistant cultures were ob- 
TABLE I

Isolations of Methicillin-Resisant Staphylococcus aureus

\begin{tabular}{cccccc}
\hline Year & Quarter & $\begin{array}{c}\text { Cultures } \\
\text { examined }\end{array}$ & Resistant* & $\begin{array}{c}\text { Laboratories } \\
\text { contributing } \\
\text { resistant } \\
\text { cultures }\end{array}$ & $\begin{array}{c}\text { Cumulative total } \\
\text { of } \\
\text { laboratories } \\
\text { contributing } \\
\text { resistant } \\
\text { cultures }\end{array}$ \\
1960 & 4 & 5440 & $3(0.06)$ & 1 & 1 \\
1961 & 1 & 4135 & 8 & 4 & 4 \\
& 2 & 3008 & $15(0.19)$ & 3 & 6 \\
1962 & 3 & 2101 & $13(0.61)$ & 6 & 13 \\
& 4 & 3708 & $11(0.30)$ & 5 & 18 \\
1963 & 1 & 3179 & $14(0.44)$ & 7 & 23 \\
& 3 & 3454 & $18(0.52)$ & 3 & 24 \\
& 4 & 2454 & $20(0.81)$ & 8 & 27 \\
1964 & 1 & 2779 & $23(0.83)$ & 8 & 30 \\
Total & 2 & 2613 & $20(0.77)$ & 10 & 35 \\
\hline & 1 & 2396 & $23(0.68)$ & 7 &. \\
\hline
\end{tabular}

*percentage in parenthesis.

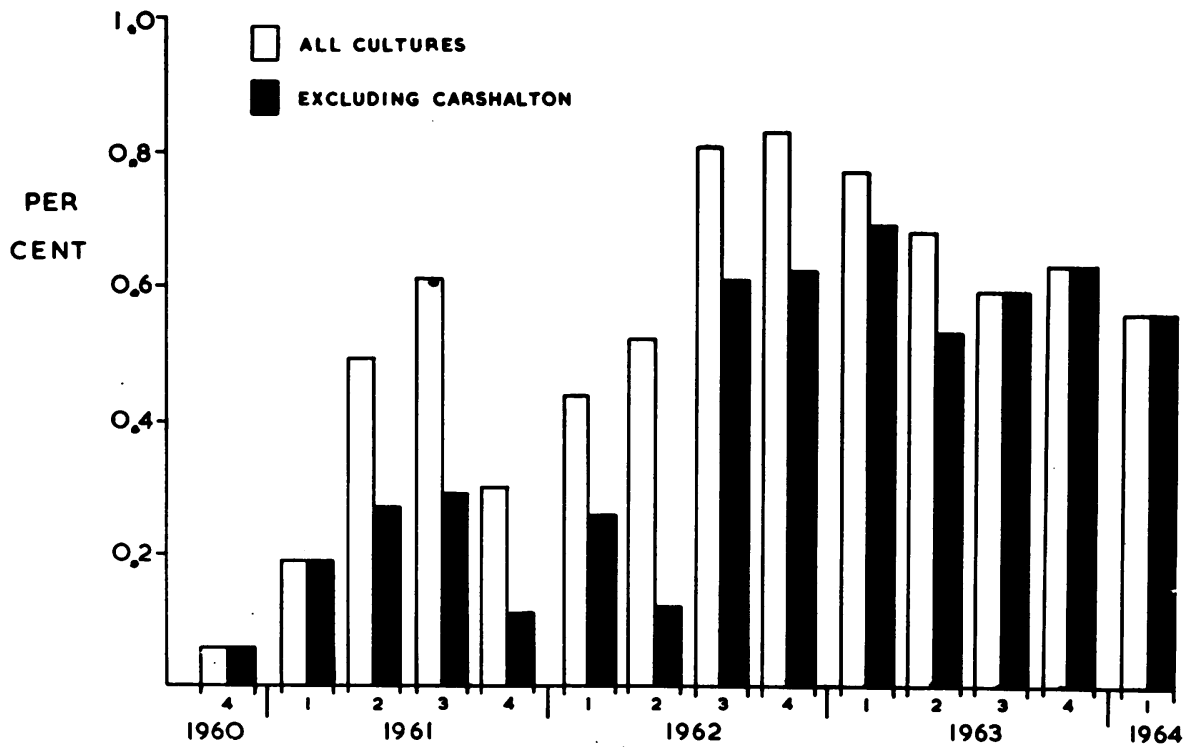

Fig. 2.-Percentage of Staph. aureus cultures resistant to methicillin in each quarter from October, 1960 to March, 1964. 
TABLE II

Source of Resistant Cultures of Staph. aureus

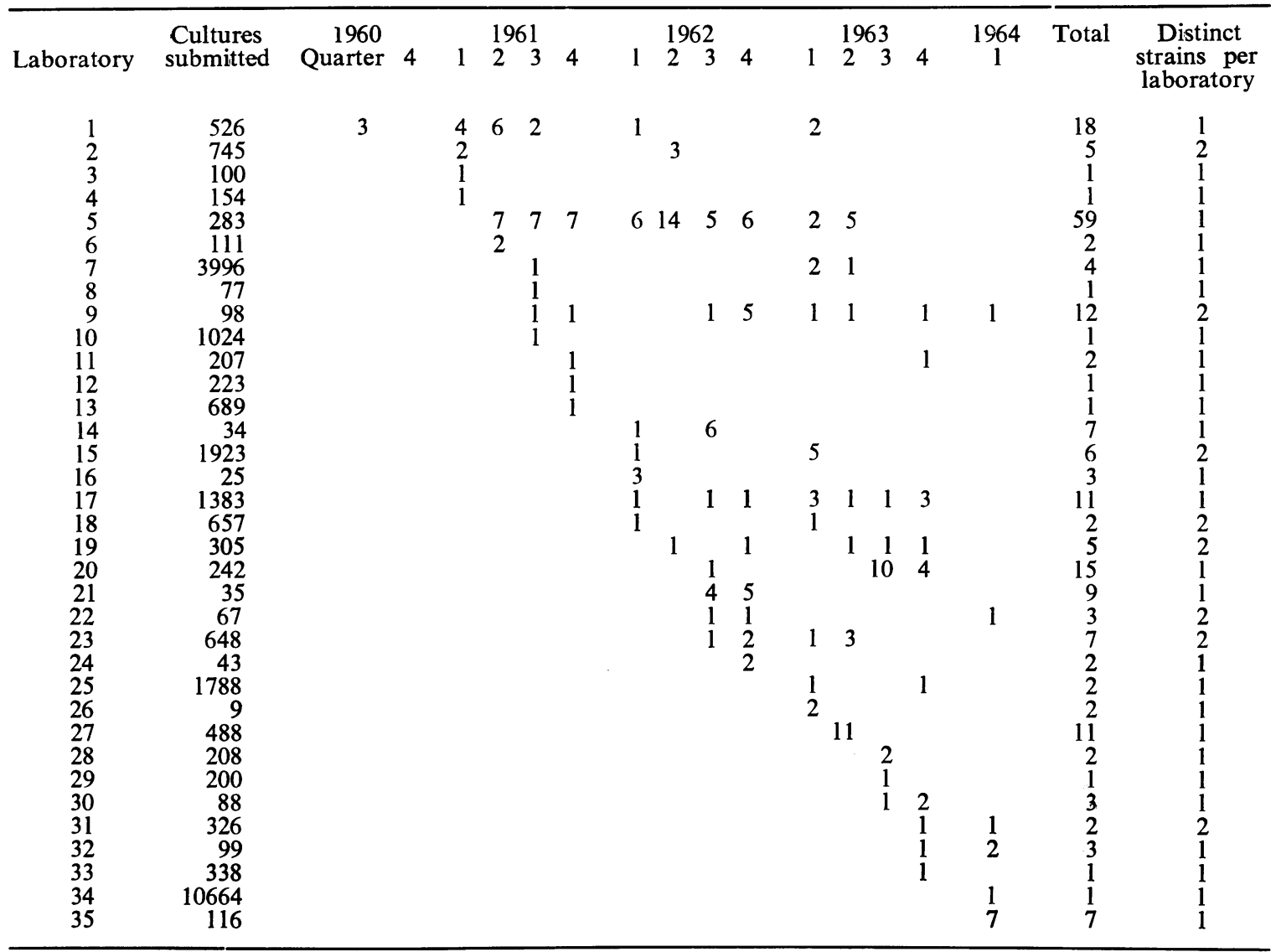

tained from any hospital sending less than ten cultures, but $10 \%$, of hospitals sending between 10 and 99 cultures, $24 \%$ of those sending 100 to 999 cultures and half of those sending 1,000 or more cultures included a resistant organism.

There were a few hospitals, however, in which a single resistant strain of Staph. aureus was endemic for long periods (e.g., Nos. 1, 5, 9, 17 and 19). No. 1 was the hospital in which a resistant organism was first identified in July 1960, and occasional isolations of the same strain were made until early 1963. Stewart and Holt (1963) have given a full account of the prevalence of a resistant strain in Queen Mary's Hospital, Carshalton (No. 5); 59 of the cultures in our series came from this source. Despite the fact that a careful search was made for infected patients in this hospital, the resistant organism was only isolated from about $2 \%$ of the patients admitted during one year.

\section{Sepsis}

Table III shows the situations in which methicillin-resistant cultures of Staph. aureus were isolated. Only one from each patient was included; when the organism was isolated both from a lesion and from a carrier site, the lesion was given as the source. Nearly half of the 213 isolations $(48 \%)$ were from lesions thought to be staphylococcal. They included three cases of septicæmia, two of meningitis, and eight lung infections confirmed post mortem. A quarter were from infected wounds.

\section{Characters}

All the resistant cultures belonged to phage 
TABLE III

Site of Isolation of 213 Methicillin-Resistant Cultures

\begin{tabular}{lrrr}
\hline Lesions & & & \\
$\quad$ Septicaemia & 3 & & \\
Meningitis & 2 & & \\
$\quad$ Lung infections & 8 & & \\
$\quad$ (post-mortem) & 6 & & \\
Abscess & 51 & & \\
Wound infection & 13 & & \\
Superficial skin lesion & 12 & & \\
Urinary infection & 102 & (48\%) \\
Other or unspecified & 7 & 34 & \\
Tputum $\quad$ Total & & 65 & \\
Carriers & & 7 & \\
Objects & & 213 & \\
Not known & & & \\
& & & \\
\end{tabular}

group III (Table IV) and nearly all of them (98\%) had one of five phage-typing patterns. Until early in 1963 the commonest pattern was $75 / 77 /+$, but most of these isolations were made in Hospital 5. In the present series, cultures with the pattern $7 / 47 / 53 / 54 / 75 / 77 /+$ were the most numerous, and were obtained from the greatest number of different foci.

The organisms described in our earlier paper (Jevons et al., 1963) as "29 (+ group III inhibitions)" have been listed in Table IV as "Untypable (+ group III inhibitions)", because they are now known to be examples of the "new" untypable staphylococcus believed to have arisen by the lysogenization of type 83A strains (see Jevons and Parker, 1964). Organisms of this sort were first observed in Britain in 1960 and have since become very widespread (Temple and Blackburn, 1963; Jacobs, Willis, Ludlam and Goodburn, 1963; Turner, 1963; Jevons and Parker, 1964). We examined large numbers of them in 1961 and 1962, but it was not until 1963 that methicillin-resistant organisms were found among them in this country. They have since become endemic in several hospitals. Similar organisms isolated in 1962 in Switzerland and South Africa were methicillin-resistant (Jevons et al., 1963).

We have recently seen two undoubted examples of naturally occurring methicillinresistant organisms which do not belong to phage group III. One was a completely untypable organism from Denmark, sent to us by Dr. K. Riewerts Eriksen, and the other was a phage group I culture (lysed by phage 29 at routine test dilution) from a British hospital. It has repeatedly been observed that when
TABLE IV

Phage-Typing Patterns of Methicillin-Resistant Staph. aureus Cultures

\begin{tabular}{|c|c|c|}
\hline Phage-typing patterns & String & Foci of \\
\hline $\begin{array}{l}7 / 47 / 53 / 54 / 75 / 77 /+ \\
75 / 77 / /+ \\
6 / 53 / 54 / 75 / 77 /+ \\
\text { Untypable }\end{array}$ & $\begin{array}{c}101 \\
68 \\
10\end{array}$ & $\begin{aligned} 20 & \stackrel{\mathrm{O}}{\mathrm{T}} \\
6 & \stackrel{\mathrm{D}}{\mathrm{D}}\end{aligned}$ \\
\hline $\begin{array}{l}83 \mathrm{~A}^{*}+\text { group III inhibition)* } \\
77 \\
42 E / 54 / 77 / 81 \\
75 \\
75 \\
7\end{array}$ & $\begin{array}{r}25 \\
4 \\
1 \\
1 \\
1 \\
1 \\
1\end{array}$ & $\begin{array}{l}5 \\
3 \\
1 \\
1 \\
1 \\
1 \\
1\end{array}$ \\
\hline
\end{tabular}
Number of hospitals from which each strain was
obtained.

*Closely related.

In italic: reactions at 1,000 times the routine test dilution

staphylococci resistant to a particular ant biotic first become prevalent they have a resf ricted range of phage-typing patterns, but that in time fresh strains with new patterns appena It will not be surprising, therefore, if methis cillin-resistant members of other phage grogipg become common in due course.

The other cultural characters of methici resistant strains of Staph. aureus are those commonly seen in hospital staphylococci. We have examined in detail 36 cultures, each representing an independent focus of infectios All were resistant to penicillin, streptomycin and tetracycline; six were resistant to chloran $\overrightarrow{\vec{F}}$ phenicol and two to erythromycin. All 36 were mercury resistant. We reported earlier (Jevor et al., 1963) that none of the first if independent cultures we tested was lipolyt for Tween 80 , but we have since seen severâ Tween-positive cultures.

Ayliffe and Barber (1963) showed that, like other strains of Staph. aureus resistant to several antibiotics, methicillin-resistant organisms usually produced large quantities of penicillinase. This was confirmed when the production of penicillinase was measured unde standard conditions (Richmond, Parker, Jevons and John, 1964). Figure 3, which is constructed from figures given by Richmond and his colo leagues (1964), shows that 20 of 23 methicillin: resistant cultures of Staph. aureus gave high yields of penicillinase (factors of 0.9 or more but not higher that those given by many othe multiple-resistant but methicillin-sensitive cul tures. 

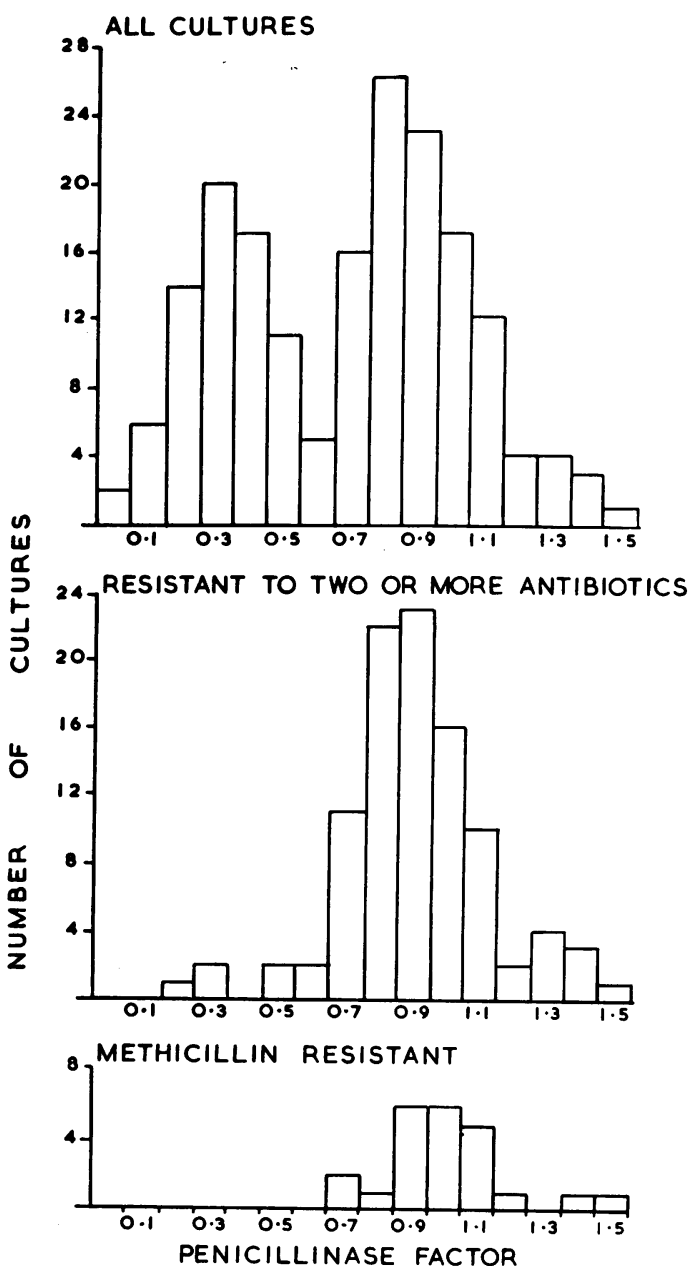

FIG. 3.-Quantity of penicillinase synthesized by cultures of Staph. aureus (from Richmond, Parker, Jevons and John, 1964).

Top: whole series of 181 cultures

Middle: 82 cultures resistant to two or more antibiotics

Bottom: 23 methicillin-resistant cultures.

A factor of 1.0 is equivalent to a penicillinase production of 330 enzyme units mg. bacteria.

\section{The significance of methicillin resistance}

There is little evidence that the use of methicillin has so far played much part in the spread of resistant strains. As far as we could ascertain, the hospitals in which resistant strains were widespread were not ones in which methicillin had been used excessively for therapeutic or prophylactic purposes. The antibiotic appears to have been used with restraint in most hospitals. Enquiries by a Public Health Laboratory Service committee in 1961-62 indicated that less than $1 \%$ of the patients in medical wards in ten provincial hospitals had received methicillin. The proportion is probably higher than this in certain special units, but methicillin appears seldom to have been used as freely as other antibiotics to which Staph. aureus has frequently been found to be resistant. There are several reports of the prophylactic use of methicillin as a spray in single hospital wards (Elek and Fleming, 1960; Ulstrup and Odegaard, 1961; Stewart, Holt and White, 1961; Goldfarb and James, 1963). In none of them was a methicillin-resistant organism isolated from any of the occupants of the ward. There is little information about the frequency with which spraying has been used as a routine procedure elsewhere, and none at all of its consequences in wards in which a resistant organism is present.

Borowski and his colleagues (1964) reported recently that over $50 \%$ of the mothers and babies in a hospital in which methicillin had never been used became colonized with a methicillin-resistant strain of Staph. aureus. This observation raises the question whether insusceptibility to methicillin might confer some other advantage on a staphylococcus. Hitherto, the accepted view has been that, in the absence of methicillin, resistance is an irrelevant or "accidental" property of strains which possess the other characters of hospital staphylococci, to which their spread in the hospital population may be attributed, for example, the active production of penicillinase and resistance to other common antibiotics.

It is well known that strains of Staph. aureus that have been rendered artificially resistant to methicillin in vitro also show an increased tolerance to benzyl penicillin (Knox and Smith, 1961; Barber, 1961). It was inferred that naturally occurring methicillin-resistant strains had the same property, but the fact that they all produced large amounts of penicillinase made this difficult to prove. We found it possible, however, to select penicillinasenegative variants from methicillin-resistant cultures by the method of Novick (1963) and investigated the action of benzyl penicillin on them. As expected, it resembled the action of methicillin on naturally methicillin-resistant staphylococci. The population in these cultures consisted of a minority of organisms with a high resistance to benzyl penicillin, and a majority with only a slightly increased resistance. In a disc sensitivity test with one unit benzyl penicillin the zone of inhibition was 
TABLE V

Sensitivity to Benzyl Penicillin of a Penicillinase-Negative Variant of a Methicillin-Resistant Culture of Staph. aureus (No. 5562) Measured by Tube-Test.

\begin{tabular}{|c|c|c|c|c|c|c|c|c|c|c|c|}
\hline \multirow[t]{2}{*}{ Culture } & \multicolumn{11}{|c|}{$\begin{array}{l}\text { Benzyl Penicillin } \\
\text { units }\end{array}$} \\
\hline & 12.5 & 6.25 & 3.12 & 1.56 & 0.78 & 0.39 & 0.19 & 0.095 & 0.05 & 0.02 & 0.01 \\
\hline $\begin{array}{l}\text { 5562: penicillinase- } \\
\text { negative variant }\end{array}$ & $\frac{-}{( \pm)}$ & $\frac{-}{(+)}$ & $\overline{(+)}$ & $\overline{(+)}$ & $\begin{array}{l}+ \\
(+)\end{array}$ & $\begin{array}{c}+ \\
(+)\end{array}$ & $\begin{array}{c}+ \\
(+)\end{array}$ & $\stackrel{+}{+}$ & $\begin{array}{c}+ \\
(+)\end{array}$ & $\begin{array}{l}+ \\
(+)\end{array}$ & $\begin{array}{c}+ \\
(+)\end{array}$ \\
\hline Oxford Staphylococous & $(-)$ & $(-)$ & $(-)$ & $(--)$ & $(-)$ & $(--)$ & $(-$ & $(-)$ & $\overline{( \pm)}$ & $\begin{array}{c} \pm \\
(+)\end{array}$ & $\begin{array}{c}+ \\
(+)\end{array}$ \\
\hline
\end{tabular}

The Oxford Staphylococcus was tested at the same time. Inoculum: 1 drop of a 6-hr. culture. At $24 \mathrm{hr} .+=$ turbidity $\pm=$ slight turbidity $-=$ no turbidity.

In parenthesis: results at $48 \mathrm{hr}$.

narrow and hazy at the edge, and with larger amounts of benzyl penicillin (three or six units) a number of discrete colonies could be seen within the zone.

The behaviour of the same cultures in a tube-sensitivity test with benzyl penicillin also resembled that seen with methicillin-resistant organisms in the presence of methicillin (Rolinson, 1961). Table $\mathrm{V}$ shows the results of an experiment in which $0.02 \mathrm{ml}$. of a six-hour broth culture was added to tubes each containing a dilution of antibiotic in one $\mathrm{ml}$. broth. The Oxford Staphylococcus had a M.I.C. of 0.05 units $/ \mathrm{ml}$. and the penicillinase-negative variant of 5562 had a M.I.C. of 1.56 units $/ \mathrm{ml}$. at 18 hours. After 48 hours, however, the methicillin-resistant organism had grown in 12.5 units of benzyl penicillin $/ \mathrm{ml}$. If the penicillin-broth mixture was incubated at $37^{\circ} \mathrm{C}$. for 18 hours before inoculation, the M.I.C. of the Oxford Staphylococcus was increased only two-fold. The M.I.C. at 18 hours for benzyl penicillin of penicillinase-negative variants of seven other methicillin-resistant organisms were between 0.09 and 3.12 units/ ml. (i.e., 2 to 64 times that of the Oxford Staphylococcus), but all showed growth in 6.25 units $/ \mathrm{ml}$. or more after 48 hours.

It is possible, therefore, that it is the natural insusceptibility of these organisms to penicillins in general, rather than their resistance to

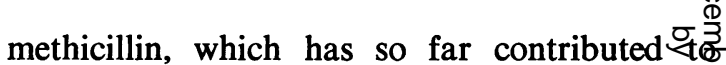
their limited success as "hospital staphylococed"을 The few individuals highly insusceptible benzyl penicillin might be able to survive andōt $\$$ multiply slowly in the presence of the antibiơtie (Sutherland and Rolinson, 1964) in environ? ments in which the number of staphylococof was not sufficient to produce an adequate cone centration of penicillinase. The fact tha insusceptibility to penicillins has so far bee found only in strains which possess severa 9 other characters common to staphylococci endemic in hospitals suggests, however, that it may not be of decisive importance fo survival and colonization. An alternative but less attractive hypothesis is that insusceptibilit to penicillins is closely linked genetically tô. some other significant character.

It is not surprising that methicillin-resistan staphylococci can, upon occasion, sprea\& widely in a hospital in which large amounts of penicillins are being used, but the frequent. persistence of a strain for long periods at a very low level of endemicity has to be explained? Many of the hospitals regularly sending us large numbers of cultures included less than one resistant culture a month, and it is a first sight difficult to see how the chain of infection was maintained. Part of the explana tion is given by the investigations of Stewart and Holt (1963). At Carshalton, most of those 
who were infected became nasal carriers but suffered from no staphylococcal illness. The first severe infection was not observed until 36 other persons had acquired the organism. In Gdańsk (Borowski et al., 1964), over 100 persons carried the organism in the nose but there was no sepsis. It seems, therefore, that in many foci of infection the nasal carriers greatly outnumber the cases of clinical infection, and unless large numbers of cultures from nasal swabs are tested for methicillin resistance the connection between successive clinical infections will be missed.

It is probable also that the disc method of screening may give us a rather low estimate of the incidence of methicillin resistance. It depends on the presence in the inoculum of individual staphylococci with a sufficiently high resistance to form discrete colonies within the zone of inhibition. As we saw in Fig. 1, the number of such individuals varies greatly. On several occasions we have seen organisms from hospitals in which a methicillin-resistant strain was endemic which had a phage-typing pattern identical with that of the resistant organism, but which appeared to be sensitive in the disc test. There have also been occasions on which the disc test suggested that an organism was resistant but the M.I.C. was less than 12.5 $\mu \mathrm{g} . / \mathrm{ml}$.

We therefore selected a group of strains with identical phage-typing patterns from one hospital, including three that were resistant and five that were sensitive to the disc test. All were resistant to penicillin and at least one other unrelated antibiotic. Two of the three apparently methicillin-resistant cultures had a M.I.C. of only $6.25 \mu$ g. methicillin $/ \mathrm{ml}$., and one of the five cultures sensitive in the disc test had a M.I.C. of $25 \mu \mathrm{g} . / \mathrm{ml}$. Penicillinase-negative variants were obtained from the five "sensitive" cultures and tested for sensitivity to benzyl penicillin. Four of them showed evidence of inherent resistance to benzyl penicillin, and had M.I.C.s of $0.39,0.39,1.56$ and 3.12 units $/ \mathrm{ml}$. respectively at 18 hours. It seems, therefore, that our definition of a methicillin-resistant Staph. aureus as an organism with a M.I.C. of at least $12.5 \mu \mathrm{g}$. methicillin $/ \mathrm{ml}$. is too strict, and that the disc test is not sensitive enough to detect all the cultures showing some natural insusceptibility to penicillins. Cultures from the same focus of infection differ considerably in the proportion of more resistant individuals they contain, and this makes it difficult to define the true extent of the spread of a resistant strain.

\section{Conclusion}

The results of our survey raise a number of difficult questions about the significance of methicillin resistance in Staph. aureus, and it is unlikely that further information collected in the same way will help much in solving them. What we now need is a series of more detailed studies in particular hospitals, in which the acquisition of resistant organisms by patients is related to the therapeutic use of various penicillins, and to the presence of antibiotic in the environment. A more suitable screening test for use in such surveys would be to inoculate heavily a single tube of broth containing perhaps $12.5 \mu \mathrm{g}$. methicillin $/ \mathrm{ml}$. and to incubate for 48 hours. (See also Sutherland and Rolinson, 1964).

\section{Summary}

Between October, 1960 and March, 1964 nearly 45,000 cultures of Staph. aureus were examined for methicillin resistance by a screening test with paper discs containing the antibiotic.

Naturally resistant organisms almost certainly existed before methicillin was synthesized in the laboratory, but have probably increased in frequency in the last few years.

Their distribution is rather patchy. occasional methicillin-resistant Staph. aureus was found in a high proportion of those hospitals from which many cultures were examined. In a few hospitals a single resistant strain became endemic and persisted for months or years. There was little evidence that this was directly related to the use of methicillin.

Nearly all the resistant cultures belonged to phage group III, and had one of a small number of phage-typing patterns. They produced large quantities of penicillinase, were resistant to mercury salts, and resistant also to one or more antibiotics unrelated to penicillin.

Methicillin-resistant staphylococci occasionally spread in hospitals in which methicillin has not been used. This directed our attention to their natural insusceptibility to benzyl penicillin. Penicillinase-negative variants of methicillin-resistant cultures consisted of a minority of individuals with a rather high resistance to benzyl penicillin, and a majority with only a slightly increased resistance. This suggested a possible mechanism for survival in the presence of penicillin under conditions in which the organisms were too few to produce an adequate concentration of penicillinase. 
A very low level of endemicity of methicillinresistant strains was observed in some hospitals. This was explained in part by the frequency of undetected symptomless infections, but there was also evidence that neither the disc sensitivity test, nor the tube sensitivity test as it is usually performed, was sufficiently sensitive to detect all resistant organisms.

\section{REFERENCES}

Ayliffe, G. A. J., and Barber, M. (1963): Brit. med. J., ii, 202.

BARBER, M. (1961): J. clin. Path., 14, 385.

BARBER, M. (1962): In "Resistance of Bacteria to the Penicillins" (edited by A. V. S. de Reuck and M. P. Cameron), p. 89. London: J. \& A. Churchill.

BoRoWSKI, J., KAMIENSKA, K., and RUTECKA, I. (1964): Brit. med. J., i, 983.

CETIN, E. T., and ANG, O. (1962): New Istanbul Contr. clin. Sci., 5, 317.

EleK, S. D., and Fleming, P. C. (1960): Lancet, ii, 569.
Goldfarb, S., and JAMES, G. C. W. (1963): Brit气

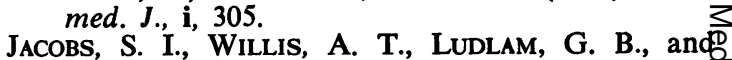
GoodBuRN, G. M. (1963): Lancet, i, 972.

Jevons, M. P. (1961): Brit. med. J., i, 124.

Jevons, M. P., COE, A. W., and PARKer, M. T $\overrightarrow{\overline{\vec{F}}}$ (1963): Lancet, i, 904.

Jevons, M. P., and PARKER, M. T. (1964): J. clin? Path., 17, 243.

KNOX, R. (1961): Brit. med. J., i, 126.

KNOX, R., and SMITH, J. T. (1961): Lancet, ii, 520.

NoviCK, R. P. (1963): J. gen. Microbiol., 33, 121.

RichMOND, M. H., PARKER, M. T., JeVONS, M. P., and JoHN, M. (1964): Lancet, i, 293.

Rolinson, G. N. (1961): Brit. med. J., i, 125.

STEWART, G. T., and HOLT, R. J. (1963): Brit. med. J., i, 308.

STEWART, G. T., Holt, R. J., and WhITE, J. A (1961): Brit. med. J., i, 863.

SUTHERLAND, R., and Rolinson, G. N. (1964): Ju

Bact., 87, 887.
TEMPLE, N. E. I., and BlaCKBURN, E. A. (1963) Lancet, i, 581.

TURNER, G. C. (1963): Lancet, i, 1156.
UlSTRUP, J. C., and ODEGAARD, A. (1961): Lancet, ii, 1227.

\title{
METHICILLIN-RESISTANT STAPHYLOCOCCI AND HOSPITAL INFECTION
}

\author{
MARY BARBER \\ Department of Bacteriology, Postgraduate Medical School of London, London, W.12
}

METHICILLIN-RESISTANT strains of Staph. aureus have now been isolated from patients in hospitals in Britain (Jevons, 1961; Barber, 1961; Knox and Smith, 1961), France (Chabbert and Baudens, 1962), Turkey (Cetin and Ang, 1962), Denmark (Eriksen and Erichsen, 1963) and Poland (Borowski, Kamienska and Rutecka, 1964). The resistance appears to be naturally occurring since in most cases the strains have been isolated from patients who have never had any of the penicillinaseresistant penicillins, and those isolated in Poland came from a hospital where the penicillinase-resistant penicillins have never been used.

In spite of the diversity of source all the recorded strains show a similar, rather unusual, type of resistance. In addition all show high penicillinase-producing activity, and multiple resistance to other antibiotics and nearly all belong to one of only a few bacteriophage types of Group III. In all cases strains resistant to methicillin show cross resistance with all the other penicillinase-resistant penicillins.

\section{Type of Resistance}

Methicillin-resistant staphylococci isolated in vitro and in clinical practice differ in the type of resistance, although in neither case do응 the strains show an increased capacity to inactive methicillin. In vitro, when methicillin-sensitive strains are passaged in medium containing methicillin they give rise to cultures which show uniform increased tolerance to the antibiotic. With penicillinase-producing organisms the resistant mutants in other respects resemble the parent cultures and the resistance $₹$ is usually stable for many generations in the 0 absence of antibiotic. With penicillin-sensitive strains, however, the resistant mutants tend to be poorly growing and relatively avirulent resembling cultures of Staph. aureus rendered ? tolerant to benzyl penicillin in vitro (Barber, N 1961).

On the other hand, naturally occurring 0 methicillin-resistant strains, when tested in the usual laboratory media, grow quite abnormally $\widetilde{\Phi}$ in the presence of methicillin, and show $a \stackrel{?}{?}$ gross difference in sensitivity according to the 0 